\title{
The Luminosity Function and Density Distribution of Disk Population Stars
}

\author{
DONNA WeIsTrop* \\ Hale Observatories, Carnegie Institution of Washington, California Institute of Technology
}

(Received 20 June 1972; revised 8 September 1972)

\begin{abstract}
The density distribution and luminosity function of the disk population are investigated, using star counts as a function of $V$ and $B-V$ for several thousand stars near the North Galactic Pole. Having estimated the contributions to the counts due to Population II stars and disk giants and subgiants, the remaining stars are assumed to constitute a pure disk-dwarf population. Density distributions for stars in successive $(B-V)_{0}$ intervals are calculated and combined to form a composite disk density distribution. The agreement with Oort's K-giant distribution is satisfactory. There is evidence that dwarfs redder than $(B-V)_{0}=1.4$ are concentrated in a narrow layer in the plane, similar to the interstellar gas. Determination of the luminosity function for the reddest disk dwarfs indicates these stars are five to ten times as numerous as was previously thought, thus accounting for a significant fraction of the missing mass in the solar neighborhood. The luminosity function is extrapolated to estimate the contribution to the missing mass of main sequence stars fainter than those considered in the analysis.
\end{abstract}

M ANY of the early studies of stars at high galactic latitude had, as their goal, the determination of the stellar density distribution perpendicular to the galactic plane (Oort 1932; van Rhijn and Schwassmann 1935 ; Becker 1940; and Bok and MacRae 1941). More recent work has continued along similar lines, with density distributions recently studied for $\mathrm{K}$ giants (Hill 1960; Oort 1960; Upgren 1962; and Elvius 1965) and main sequence stars of spectral types A-G (Upgren 1962, 1963; and Elvius 1965). These investigations, like earlier ones employing spectral classifications, were limited to stars brighter than photographic magnitude 13. Only Fenkart (1967) has investigated the distributions for fainter stars, but his results are limited to stars with absolute magnitude $M_{\mathrm{G}}=3-8$. A study of colormagnitude data for faint disk stars at high galactic latitudes is therefore of considerable interest for determining the disk density distribution far from the plane, and also a method of deriving the luminosity function in the plane for intrinsically faint stars. No recent determination of the latter has been made, McCuskey's extensive work being limited to stars of absolute photographic magnitude 7 and brighter (McCuskey 1956 and references therein). For fainter stars, the early work of van Rhijn (1936) and Luyten (1938) must be used, so that it seems worthwhile to rederive the luminosity function using data now available.

In a previous paper (Weistrop 1972, henceforth referred to as Paper I), an upper limit to the local Population II density has been derived, using star counts as a function of color and apparent visual magnitude. In the current work, the data is used to determine the density distribution and luminosity function for disk stars. The observations are discussed in Paper I. They consist of $B$ and $V$ magnitudes for 13820 stars in an area of $13.5 \mathrm{sq}$ deg centered on S.A.57. The magnitudes were determined from iris photometry of plates taken with the 48-inch Schmidt telescope on Palomar Mountain. The Population II component of the star

* Present address: Department of Physics and Astronomy, Tel Aviv University, Tel Aviv, Israel. counts has been estimated for each of the eight models described in Paper I. The models will be referred to by giving the name of the globular cluster used to represent the Population II luminosity function (M3 or M92), the value assumed for $n$ (the Population II density in the plane is assumed to vary with distance from the galactic center, $\mathrm{R}$, as $\mathrm{R}^{-n}$, where $n=3$ or 4 ), and the axial ratio $(1: 1$ or $1: 2)$ of the ellipsoids of constant density in which Population II stars are assumed to be distributed. All stars not belonging to Population II are assumed to be members of the disk population.

The number of disk members expected to be giants or subgiants is estimated in Sec. I. Dwarf density distributions are derived as a function of color and combined to determine a general disk density distribution (Sec. II). Some evidence is presented indicating the density distribution near the plane for the faintest stars is steeper than was previously thought. The disk luminosity function and local stellar mass density are discussed in Sec. III. In the fourth section, the results are compared with those of other authors. The composite disk density distribution is found to be in satisfactory agreement with distributions derived by Oort (1960) and Fenkart (1967). Alternate explanations for the increased luminosity function derived for intrinsically faint stars are considered and rejected, and the consequences of this luminosity function for the local mass density are discussed. Part of the missing mass in the galactic plane can be accounted for by the increased number of intrinsically faint stars.

\section{GIANTS AND SUBGIANTS}

Before the dwarf density functions can be derived, some estimate of the number of giants included in the disk star counts must be made. To calculate the number of giants we require values for several parameters: the absolute magnitude of the giants as a function of $B-V$, their relative number distribution as a function of $B-V$, the relative density distribution of the giants, and a constant factor which normalizes the computed number 
TABLE I(a). Absolute magnitude calibration and relative color distribution for subgiants.

\begin{tabular}{cccccc}
\hline \hline & & \multicolumn{4}{c}{ Rel. No. } \\
$(B-V)_{o}$ & $M_{V}$ & NGC 6791 & M67 & NGC 188 & Adopted \\
\hline 0.55 & 3.00 & 0.00 & 0.38 & 0.00 & 0.08 \\
0.65 & 3.50 & 0.16 & 0.28 & 0.17 & 0.19 \\
0.75 & 3.50 & 0.30 & 0.10 & 0.07 & 0.17 \\
0.85 & 3.50 & 0.09 & 0.13 & 0.06 & 0.08 \\
\hline \hline
\end{tabular}

TABLE I(b). Absolute magnitude calibration and relative color distribution for giants.

\begin{tabular}{ccc}
\hline \hline$(B-V)_{o}$ & $M_{V}$ & Rel. No. \\
\hline 0.95 & 0.60 & 0.26 \\
1.05 & 0.80 & 0.30 \\
1.15 & 0.80 & 0.23 \\
1.25 & 0.50 & 0.08 \\
1.35 & 0.00 & 0.04 \\
1.45 & -0.20 & 0.04 \\
1.55 & -0.30 & 0.04 \\
1.65 & -0.40 & 0.01 \\
\hline
\end{tabular}

of giants to the observed number for an interval in $B-V$ and $V$ for which observations are available. The parameters are used in the equation of stellar statistics (Sec. II) to predict the number of giants in each colorapparent magnitude interval of interest. Absolute magnitudes for giants (luminosity class III) as a function of spectral type are given by Blaauw (1963). The relation between spectral type and color was calibrated using data for luminosity class III stars in the Catalogue of Bright Stars (Hoffleit 1964, hereinafter referred to as BSC). The results agree quite satisfactorily with those of Johnson and Morgan (1953). The adopted absolute magnitude-color relation for the giants is shown in Table I(b).

The absolute magnitudes in Table $\mathrm{I}(\mathrm{b})$ were derived from stars selected according to apparent magnitude. For the current calculation, however, absolute magnitudes derived from stars selected per unit volume of space are required, as the magnitudes are considered characteristic of stars in a limited volume of space. In order to transform the magnitudes in Table I to the required form, Eq. (1) is used

$$
M_{m}=M_{o}-\frac{\sigma^{2}}{\operatorname{Mod}} \frac{d \log A(m)}{d m},
$$

where $M_{m}$ is the absolute magnitude for stars selected by apparent magnitude; $M_{o}$, the absolute magnitude for stars selected per unit volume; $A(m)$, the number of stars at $m$; and $\sigma$, the dispersion of the absolute magnitudes around $M_{o}$ (Malmquist 1936). A value of 0.60 mag was adopted for $\sigma$, determined by fitting Gaussians to the luminosity functions of late $\mathrm{G}$ and early $\mathrm{K}$ giants as determined by Halliday (1955) and Sandage (1957). A value of 0.2 was adopted for $d \log A(m) / d m$, from the star counts of the present study. This value must be considered only an estimate and was adopted in the absence of available data for giants fainter than $B=13.0$. The data which exist for brighter giants do not contradict this assumption. Oort (1960) indicates $d \log A(m) / d m \approx 0.15$ for $m_{p g}=11.0-13.0$. From Upgren's (1962) smoothed $A(m)$ for giants, we find $d \log A(m) / d m$ decreasing from 0.38 at $B=8.0$ to 0.20 at $B=11.5$. Beyond $B=12.0$, the number of giants counted actually decreases, but this may be due in part to omissions in the catalogue, although Upgren states that the catalogue is complete to $m_{p g}=13.0$. In view of these data and the results from the star counts presented here, the value $d \log A(m) / d m=0.2$ was adopted for the giants.

The color distribution of the giants was determined by counting the number of luminosity class III stars in the BSC in $B-V$ intervals of $0.10 \mathrm{mag}$. Because the BSC has a limiting magnitude of $V=6.5$, the volume of space surveyed for a particular type of star depends on the absolute magnitude. Using the absolute magnitudes adopted above, the volume surveyed for each group of giants was determined and the counts reduced to a standard volume. The total number of giants was then normalized to 1.00 [Table I(b)]. Since the data from the BSC is used only to determine the relative color distribution of the giants, errors resulting from systematic misidentification of giants or the selective omission of giants of a specific color would change the calculated color distribution but not the total number predicted for a given apparent magnitude. As can be seen from Table II, the total number of giants calculated for all apparent magnitudes is quite small, a total of 8.5 stars. Therefore, any error introduced by selective omission or misidentification of giants as a function of color has a negligible effect on the results. For the relative density distribution of the giants, an exponential approximation to Oort's curve for K giants (1960) was adopted. Using these parameters and a total absorption in $V$ of $0.10 \mathrm{mag}$ (see Paper I), the number of $\mathrm{K}$ giants between $V=11$ and 12 was estimated. This number was normalized to 25 , the number of $K$ giants per magnitude at $V=11.5$ for the area of the sky investigated here, 13.5 sq deg (Oort 1960; Hill 1960). Using the normalization factor, the number of giants as a function of $(B-V)_{o}$ and $V$ for $V=12-18$ was estimated (Table II). The predicted number of giants was subtracted from the disk star counts, which had previously been corrected for errors in $B-V$ (assuming a gaussian error function with $\sigma=0.15 \mathrm{mag}$ ) and the adopted color excess, $E(B-V)=0.03 \mathrm{mag}$ (Paper I).

A procedure similar to that used for the giants was adopted to predict the contribution of the subgiants to the disk star counts. Values for the absolute magnitudes and color distribution of the subgiants were adopted, and assumptions made concerning the number of subgiants with respect to dwarfs and the density distribution of these stars. The parameters were then used in the equation of stellar statistics to predict the number of subgiants as a function of color and apparent magni- 
tude. Since little is known about the distribution and total number of field subgiants, we have assumed for the present calculation that the absolute magnitudes and color distribution of these stars can be represented by those for the subgiants in the old galactic clusters NGC 6791 (Kinman 1965), M67 (Johnson and Sandage 1955 ; Eggen and Sandage 1964), and NGC 188 (Sandage 1962; Eggen and Sandage 1969). The absolute magnitudes are determined from the mean of the absolute magnitudes of the cluster subgiants in the $(B-V)_{0}$ interval of interest, rounded to the nearest $0.25 \mathrm{mag}$, and correspond to magnitudes for stars selected per unit volume of space. Stellar evolution studies indicate that subgiants come from that part of the main sequence just above the current turn-off point. It therefore seems reasonable to relate the number of subgiants to the number of stars still on the main sequence but close to the turn-off point. The number of subgiants in each $(B-V)_{o}$ interval was determined relative to the number of dwarfs with $(B-V)_{o}=0.6-0.7$ from star counts for the three clusters. It was assumed that, at any $z$, the relative number of field subgiants in each $(B-V)_{o}$ interval to dwarfs between $(B-V)_{o}=0.6-0.7$ remained constant at this value. This assumption is made due to the absence of any information concerning the change in density of subgiants with distance from the galactic plane. No conclusions can be drawn from the ratios for the individual clusters. From Kinman, the distances above the galactic plane are $z=1 \mathrm{kpc}, 0.4 \mathrm{kpc}$, and $0.5 \mathrm{kpc}$ for NGC 6791, M67, and NGC 188, respectively. Yet, the ratios of subgiants to dwarfs derived for NGC 188 are very similar to those for NGC 6791, while the ratios for M67 and NGC 188 are dissimilar [Table $\mathrm{I}(\mathrm{a})]$. Clearly, distance above the galactic plane is not the determining factor for the subgiantdwarf ratios in these clusters. Therefore, in the absence of other information, it is assumed that the subgiant density distribution varies in the same way as the density distribution of the stars from which they evolve. Since the field stars may be expected to have a range in age, as well as other parameters, we combine the results for the three clusters to determine the ratios to be used for the field population. The adopted ratios, as well as the absolute magnitudes, are shown in Table I(a). Using these assumptions, the relative density distribution of the subgiants is the same as that for the dwarfs with $(B-V)_{o}=0.6-0.7$.

An iterative technique was used to estimate the number of subgiants in the disk star counts. As a first approximation, all the disk stars between $(B-V)_{o}=0.6$ and 0.7 were assumed to be dwarfs, and the density distribution of these stars was derived as described in the next section. With the density distribution and parameters previously described, the number of subgiants with $(B-V)_{o}=0.6-0.7$ was estimated for $V=12-18$. These numbers were then subtracted from the total number of stars at the corresponding magnitude, and the remaining stars used to determine a new
TABLE II. Estimated number of giants and subgiants.

\begin{tabular}{|c|c|c|c|c|c|c|}
\hline$V$ & 12.5 & 13.5 & 14.5 & 15.5 & 16.5 & 17.5 \\
\hline \multicolumn{7}{|c|}{ Giants } \\
\hline $\begin{array}{c}(B-V) o \\
0.95\end{array}$ & 1.8 & 0.1 & $\ldots$ & $\ldots$ & $\ldots$ & $\ldots$ \\
\hline 1.05 & 3.3 & 0.2 & $\ldots$ & $\ldots$ & $\ldots$ & $\cdots$ \\
\hline 1.15 & 2.5 & 0.1 & $\cdots$ & $\cdots$ & $\cdots$ & $\ldots$ \\
\hline 1.25 & 0.5 & $\ldots$ & $\ldots$ & $\cdots$ & $\ldots$ & $\ldots$ \\
\hline 1.35 & $\cdots$ & $\cdots$ & $\cdots$ & $\ldots$ & $\ldots$ & $\ldots$ \\
\hline 1.45 & $\ldots$ & $\ldots$ & $\cdots$ & $\cdots$ & $\ldots$ & $\ldots$ \\
\hline 1.55 & $\cdots$ & $\cdots$ & $\cdots$ & $\cdots$ & $\cdots$ & $\cdots$ \\
\hline 1.65 & $\cdots$ & $\cdots$ & $\cdots$ & $\cdots$ & $\cdots$ & $\cdots$ \\
\hline \multicolumn{7}{|c|}{ Subgiants } \\
\hline 0.55 & 6.9 & 8.4 & 9.4 & 2.0 & 0.1 & $\ldots$ \\
\hline 0.65 & 15.2 & 18.6 & 21.8 & 13.2 & 1.1 & $\ldots$ \\
\hline 0.75 & 13.6 & 16.6 & 19.6 & 11.8 & 1.0 & $\ldots$ \\
\hline 0.85 & 6.4 & 7.8 & 9.2 & 5.6 & 0.5 & $\ldots$ \\
\hline
\end{tabular}

dwarf density distribution. The procedure was iterated until the newly calculated dwarf distribution was the same as the assumed one; five iterations generally being sufficient to obtain convergence. Having determined the dwarf density distribution at $(B-V)_{o}=0.6-0.7$, the number of subgiants in each $(B-V)_{o}$ interval and at each apparent magnitude was calculated (typical estimates are shown in Table II). The predicted number of subgiants was subtracted from the star counts, and all remaining stars assumed to be dwarfs.

\section{DENSITY DISTRIBUTION}

The absolute magnitude calibration for the dwarfs bluer than $(B-V)_{o}=1.40$ was adopted from Blaauw, using the relation between color and spectral type given by Johnson and Morgan (1953). For redder stars, the absolute magnitudes were determined from data given by Eggen (1968) by averaging the absolute magnitudes for all stars with $(B-V)_{o}$ within 0.10 mag intervals. This calibration is consistent with recent color-magnitude relations determined for nearby stars by Woolley, Epps, Penston, and Pocock (1970) and Strand and Riddle (1970) (Table III). The absolute magnitude calibration for all dwarfs is given in Table IV. These magnitudes, like those for the giants previously discussed, are for stars selected according to apparent magnitude. An absolute magnitude dispersion of $0.30 \mathrm{mag}$ was adopted for all stars bluer than $(B-V)_{o}=1.40$ (Gliese 1956). For stars redder than $(B-V)_{o}=1.40$, the absolute magnitude dispersion was assumed to be $0.75 \mathrm{mag}$. This dispersion is an estimate which reflects the change in $M_{V}$ over an interval of $0.10 \mathrm{mag}$ in $(B-V)_{o}$, about $1.5 \mathrm{mag}$ for these stars (Table IV), as well as the scatter around the adopted color-magnitude relation of the stars used in the calibration.

Density distributions were calculated for stars in intervals of $0.10 \mathrm{mag}$ in $(B-V)_{o}$ for $(B-V)_{o}=0.40-1.70$, using the equation of stellar statistics [Eq. (2)], where $A(m)$ is the number of stars between apparent magnitudes $m-\frac{1}{2}$ and $m+\frac{1}{2}, \omega$ is the area of the sky over which 
TABLE III. Comparison of absolute magnitude calibrations for reddest stars.

\begin{tabular}{crrr}
\hline \hline$B-V$ & $M_{V^{\mathrm{a}}}$ & $M_{V}{ }^{\mathrm{b}}$ & $M_{V^{c}}$ \\
\hline 1.35 & 8.2 & & 8.1 \\
1.45 & 9.0 & 10.5 & 9.6 \\
1.55 & 11.3 & 11.4 & 11.1 \\
1.65 & 12.4 & 12.4 & 12.6 \\
\hline
\end{tabular}

a Wooley et al. (1970).

b Strand and Riddle (1970).

${ }^{c}$ Eggen (1968).

the stars were counted $\left(4.11 \times 10^{-3}\right.$ steradian for this investigation), $D(z, M)$ is the number of stars per cubic parsec with absolute magnitude $M$ at height $z$ above the plane, and $z^{2} d z$ is the volume element

$$
A(m)=\omega \int_{0}^{\infty} D(z, M) z^{2} d z .
$$

An initial density distribution was established by assuming that the dispersion in absolute magnitude is zero. Then, using the absolute magnitudes from Table IV, the volume occupied by stars of given absolute magnitude for each interval of apparent magnitude was calculated. The first approximation to the density distribution was found by dividing the observed star counts for each apparent magnitude interval by the corresponding volume. Using this distribution, the number of stars, $A\left[m,(B-V)_{o}\right]$ was predicted from Eq. (2), now assuming the absolute magnitude dispersions given above. The results were then compared with the dwarf star counts. Generally, for stars in a given $(B-V)_{0}$ interval, the total number of stars predicted was about the same as the total number observed, but the distribution with apparent magnitude was altered, some of the predicted counts being larger than the observed counts, and others smaller. Except for those intervals containing very few stars, the agreement between this first set of predicted counts and the observed counts was within $25 \%$. The assumed density distribution was then corrected by the ratio of the observed counts to predicted counts as a function of apparent magnitude. The procedure was repeated with the new density distribution until convergence was obtained, ten iterations being sufficient for all but the reddest color intervals, which required 15 . In most cases, the agreement between computed and observed counts was well within the square root of the counts, the poorer results coming for intervals containing few stars.

The density distributions were derived for the number of disk dwarfs predicted by each of the Population II models. The results for $(B-V)_{0}$ intervals centered at $0.45-0.85$ are shown for three models in Figs. 1(a)-1(c). The most striking feature of the diagrams is the lack of internal consistency for the density distributions derived using model M3, $n=3,1: 1$. As can be seen from Fig. 1(a), the model indicates stars with colors $(B-V)_{o}=0.4-0.7$ exist at distances of $2 \mathrm{kpc}$ from the galactic plane, but stars with $(B-V)_{0}=0.8-0.9$ disappear beyond $800 \mathrm{pc}$. Nothing in our present knowledge of stellar distributions would explain such a result. Inconsistencies of this sort are found for all M3 models except $n=4,1: 2$. The M92 models are all internally consistent except for model $n=3,1: 1$; although there is some indication that the density distributions for M92, $n=4,1: 2$ most clearly define a common density distribution. The difference in internal consistency for the M3 and M92 models is due to the difference between the luminosity functions of the clusters, and may imply that the M92 luminosity function better represents the field Population II than does the M3 luminosity function. Thus, the results seem to indicate that the M92, $n=4,1: 2$ model is most representative of the field Population II and all subsequent results will be given for this model only. The density distributions calculated under the assumption of no Population II stars are shown in Fig. 2 for comparison. Their similarity to the distributions for the preferred model indicates the small degree to which the assumptions about Population II influence the overall results for the disk population.

The similarity of the density distribution for stars of various colors [Figs. 1(c), 3 and 4 ] suggests the possibility of determining a well-defined composite disk density distribution from the individual curves. To determine the composite distribution, intervals were established in $z$ with widths corresponding to $\Delta m=0.50$ mag. The average difference in $\log D(z)$ for adjacent intervals was determined using only those individual density curves defined in both intervals. For this calculation, all densities determined from counts for which the estimated giant or subgiant contribution was one third or more were omitted. Because of the uncertainty in the giant and subgiant calculations, those densities derived from counts for which dwarfs were not the major contributor were considered unreliable. The relative densities at a given height above the plane were used to weight the individual curves. The weights were determined by fitting the individual density curves to the composite curve of the previous iteration. The succession of average density ratios just defined the new

TABLE IV. Absolute magnitude calibration for disk dwarfs.

\begin{tabular}{cc}
\hline$(B-V)_{o}$ & $M_{V}$ \\
\hline 0.45 & 3.30 \\
0.55 & 4.05 \\
0.65 & 4.80 \\
0.75 & 5.60 \\
0.85 & 6.00 \\
0.95 & 6.35 \\
1.05 & 6.65 \\
1.15 & 7.05 \\
1.25 & 7.50 \\
1.35 & 8.10 \\
1.45 & 9.60 \\
1.55 & 11.10 \\
1.65 & 12.60 \\
\hline
\end{tabular}




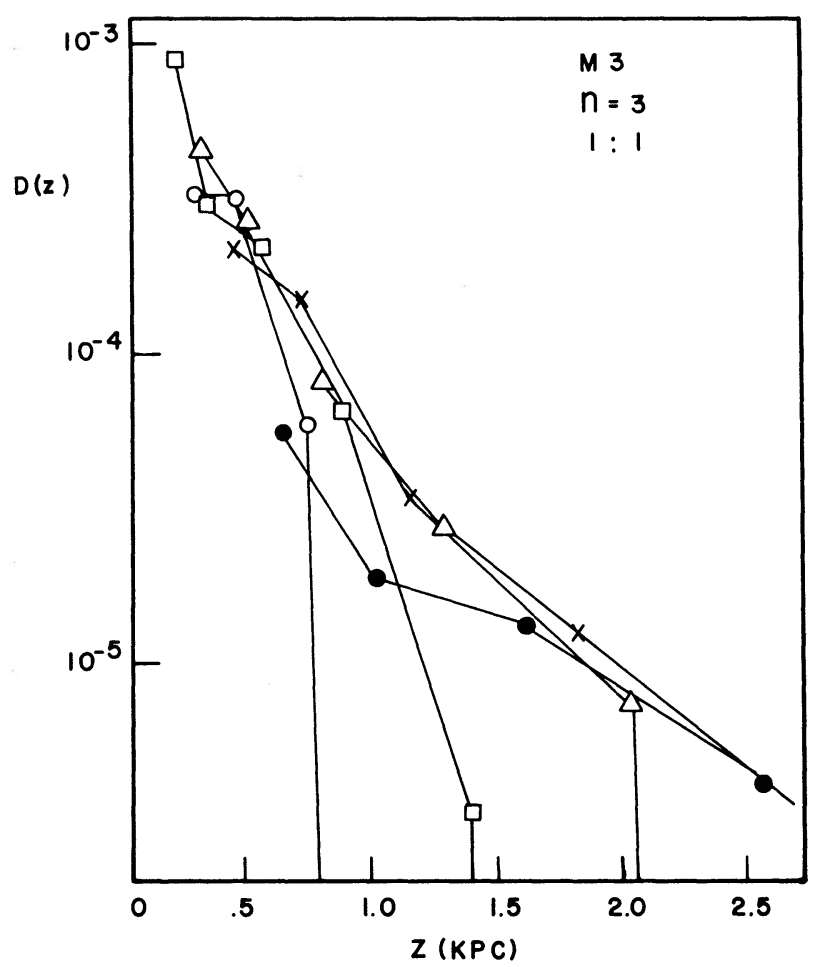

(a)

Fig. $1(\mathrm{a}-\mathrm{c})$. Density distributions for disk dwarfs calculated for three Population II models. Individual curves are shown for $(B-V)_{o}=0.4-0.5(\bullet), 0.5-0.6(\times), 0.6-0.7(\triangle), 0.7-0.8(\square)$, and $0.8-0.9(0)$.

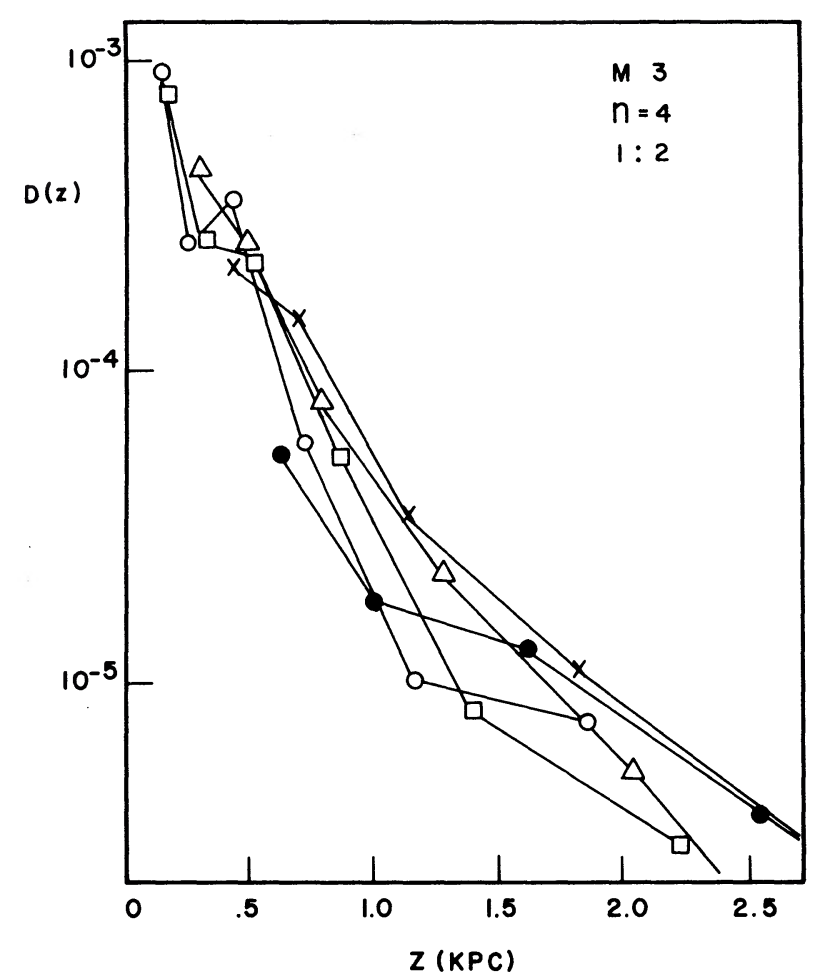

(b)

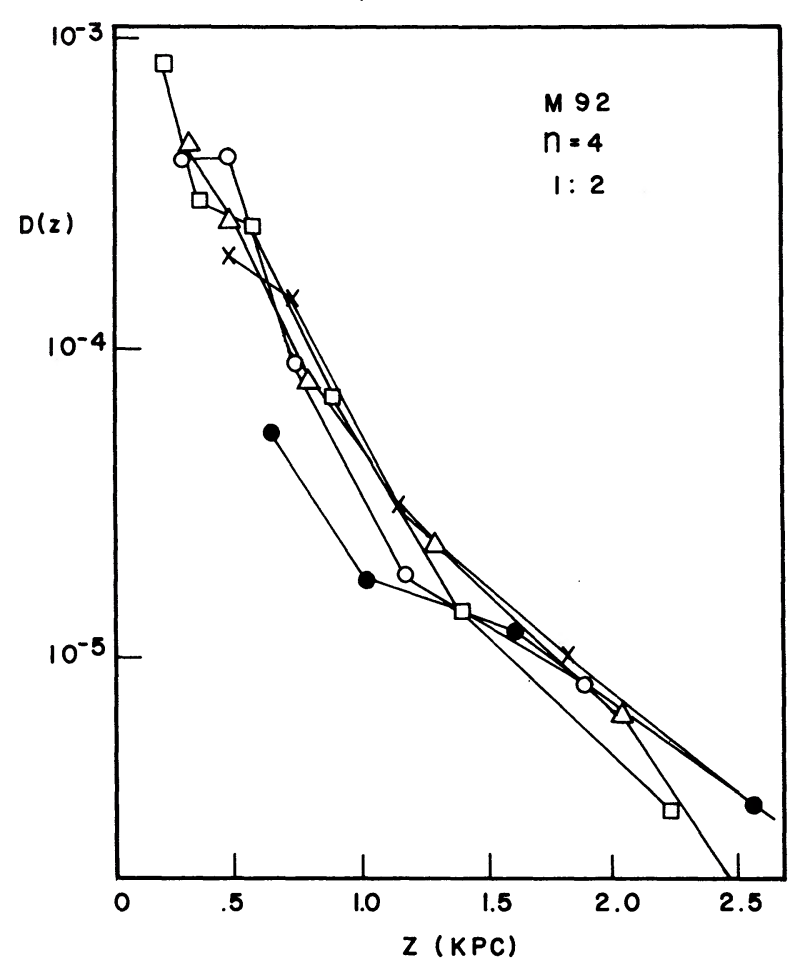

(c)

composite curve. Having established a composite curve, new weights for the individual density curves were determined, and the process was iterated until the value of the weights converged. Ten iterations were more than sufficient in all cases. The composite curve for the M92, $n=4,1: 2$ model is shown in Fig. 5. The innermost points are not indicated in the figure, owing to its reduced horizontal scale. All values for the indi- 


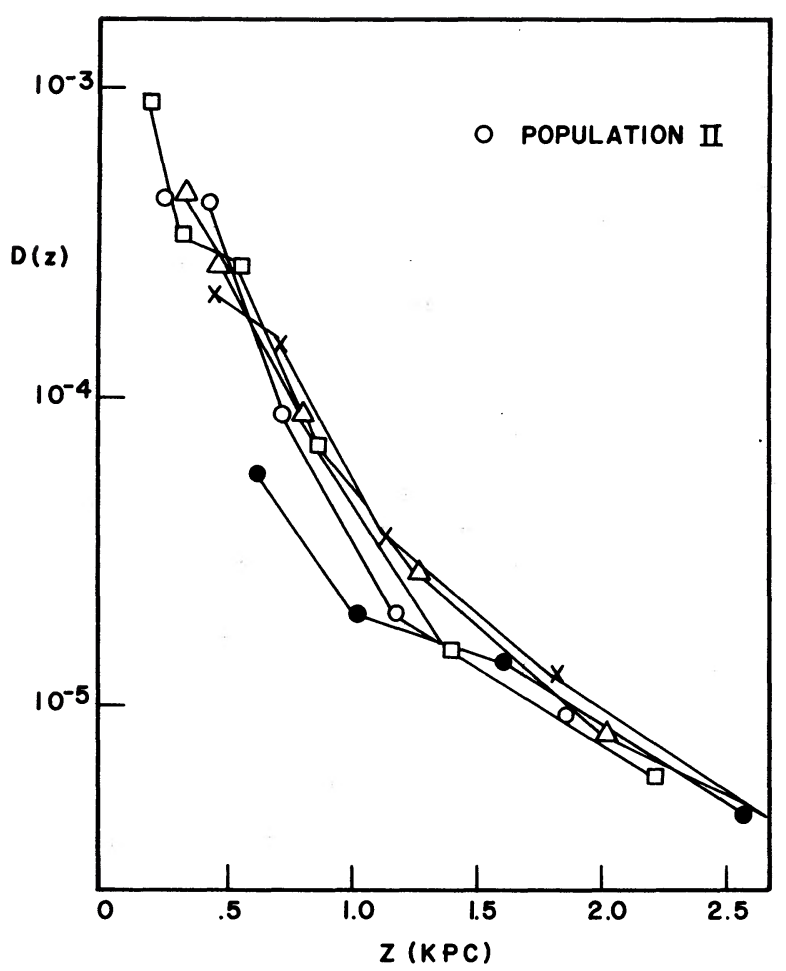

Fig. 2. Density distributions for disk dwarfs calculated under the assumption of no Population II contribution to the star counts. Symbols are the same as Fig. 1.

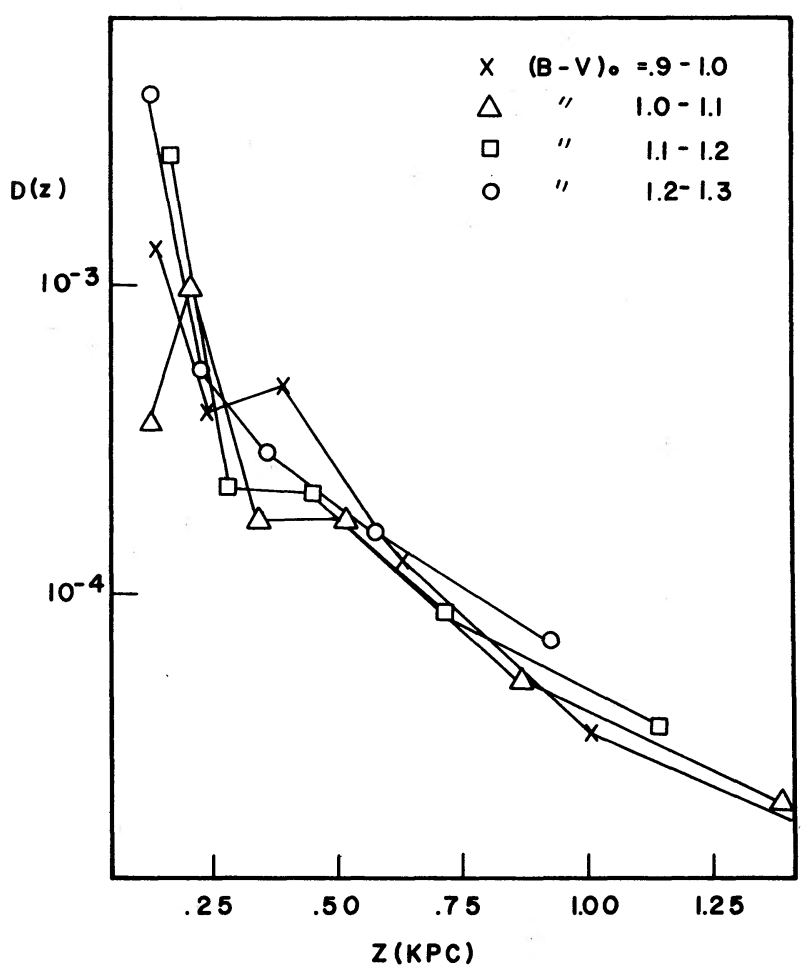

Fig. 3. Density distributions of red disk dwarfs calculated for model M92, $n=4,1: 2$.

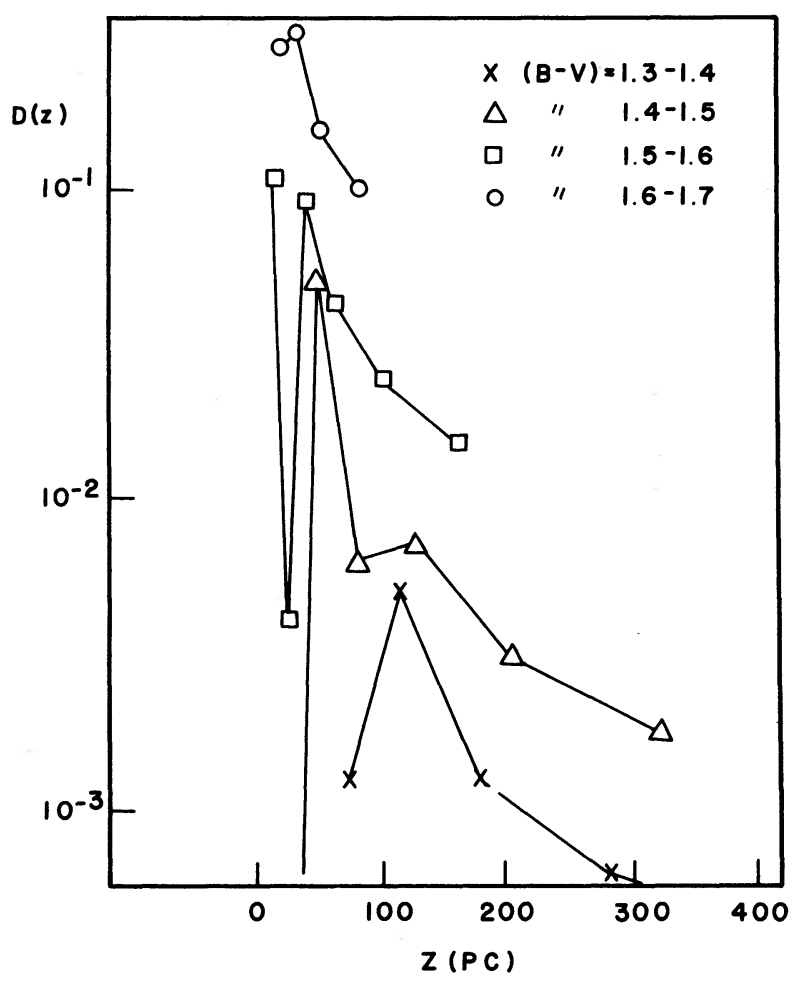

FIG. 4. Density distributions of very red disk dwarfs calculated for model M92, $n=4,1: 2$.

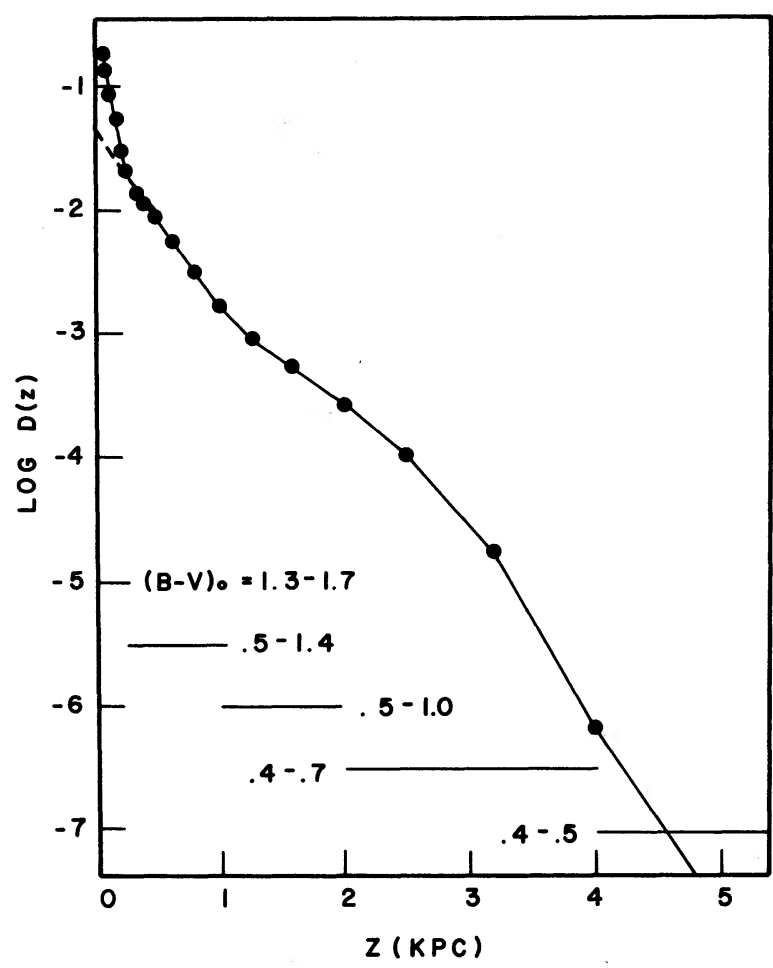

FIG. 5. Composite disk density distribution. The dashed line indicates the exponential extrapolation to the plane discussed in the text. The $(B-V)_{o}$ colors of the stars determining each part of the curve are indicated. 
vidual points defining the curve are given in Table $\mathrm{V}$. The results beyond $3 \mathrm{kpc}$ are tentative, as only the bluest disk stars studied penetrate to these distances, and these are most effected by the Population II assumptions.

As can be seen from Fig. 5, the composite density distribution indicates a very steep density gradient within $200 \mathrm{pc}$ of the plane, a result contrary to previous determinations. Because of the apparent magnitude limits of the observed material, the composite curve at small $z$ is determined by the individual density curves for the reddest dwarfs (Fig. 5). Therefore, in order to check the validity of the steep density gradient at small distances from the plane, an attempt was made to reproduce the counts of the reddest stars assuming a more conventional density distribution. To make this calculation, the composite curve was extrapolated to $z=0$ as indicated by the dashed line in Fig. 5, which is equivalent to assuming the density distribution is exponential close to the plane. This new density distribution was then used to predict the star counts in question, using Eq. (2). This calculation, using the absolute magnitudes, dispersion in absolute magnitude and absorption adopted previously, did not reproduce the observed counts, except for the stars with $(B-V)_{o}$ $=1.30-1.40$. The observed counts for these stars were adequately represented by the extrapolated density distribution and other previously adopted parameters, and so no stars bluer than $(B-V)_{o}=1.40$ will be considered in the following discussion. Attempts were also made to reproduce the observed counts by using the extrapolated density curve and adopting new values for the absolute magnitude dispersion. Typical examples of these predictions may be seen in Fig. 6, along with the star counts to be fit, for stars with $(B-V)_{o}=1.40$ 1.50. Only for a dispersion of $2.00 \mathrm{mag}$ were the fits

TABLE V. Composite density distribution.

\begin{aligned} & \hline \hline$z(\mathrm{pc}) \log D(z) \\ &$\hline 25-0.8975 \\ & 32-0.8176 \\ & 40-0.7371 \\ & 50-0.7323 \\ & 63-0.8461 \\ & 80-0.9932 \\ & 100-1.0671 \\ & 126-1.1184 \\ & 158-1.4943 \\ & 200-1.6938 \\ & 250-1.8693 \\ & 320-1.9379 \\ & 400-2.0512 \\ & 500-2.2434 \\ & 630-2.4980 \\ & 800-2.7654 \\ & 1000-3.0254 \\ & 1260-3.2480 \\ & 1580-3.5400 \\ & 2000-3.9469 \\ & 2500-4.7452 \\ & 3200-6.1507 \\ & 4000 \\ & \hline\end{aligned}

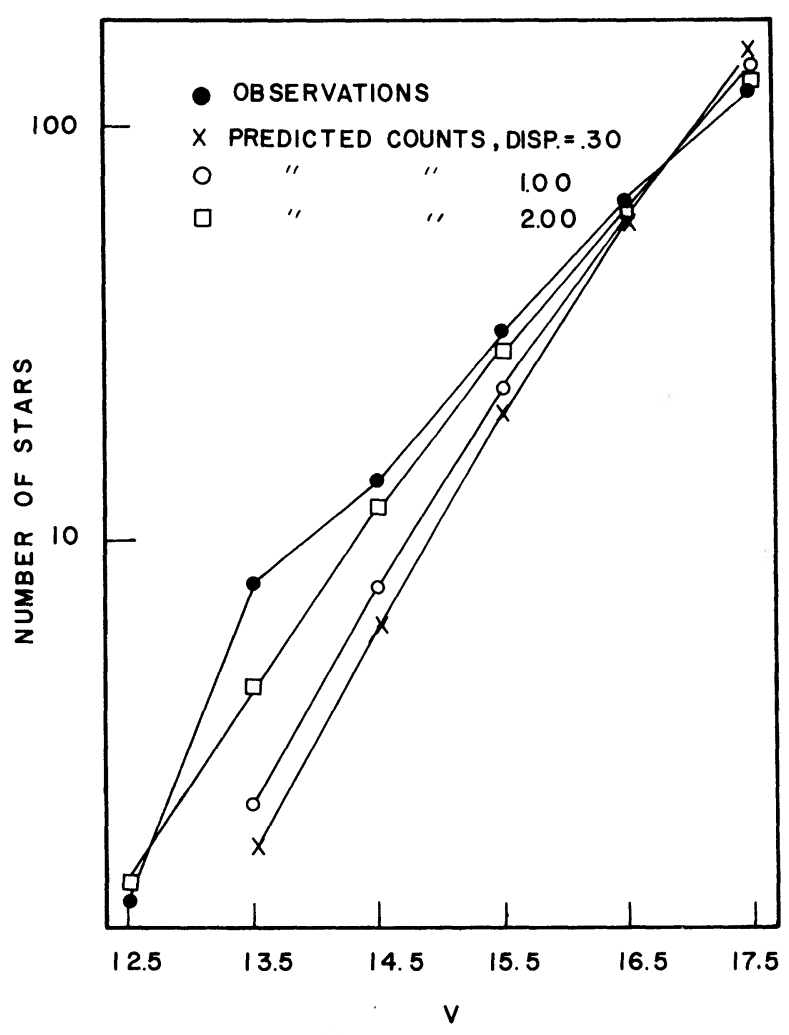

FIG. 6. Predicted star counts as a function of absolute magnitude dispersion for stars with $(B-V)_{o}=1.4-1.5$.

satisfactory, except for the stars for which $(B-V)_{o}$ $=1.60-1.70$, which could not be fit even with this large dispersion. Yet, an examination of Eggen's data (1968) indicates the scatter around the adopted $M_{V^{-}}(B-V)_{0}$ relation corresponds to $\sigma=0.6 \mathrm{mag}$ precluding a dispersion of 2.00 mag. Finally, an attempt was made to fit the observed counts by increasing the assumed interstellar absorption to $0.50 \mathrm{mag}$, but the slope of the observed relation between star counts and apparent magnitude was not reproduced. The only alternative, therefore, is the acceptance of the steep $D(z)$ determined by the data.

A composite density distribution was derived using only the data for stars with $(B-V)_{0}$ redder than 1.40 . The individual points calculated are shown in Fig. 7, along with the smoothed curve hand-fitted to them. The smoothed curve was fitted to the extrapolated density distribution of Fig. 5, so that for small $z$ the curve is determined by the composite for the red stars, while for large $z$ the shape of the curve is the same as that derived for bluer stars. Because the existence of a steep density distribution close to the plane must be considered controversial, three possible stellar density distributions will be considered in the remaining analysis. For the first, Case I, we assume the true density distribution for all disk stars is that given by the dashed curve in Fig. 7 normalized to $\log D(z)=0.00$ at $z=0$, which is the same as the extrapolated curve of 


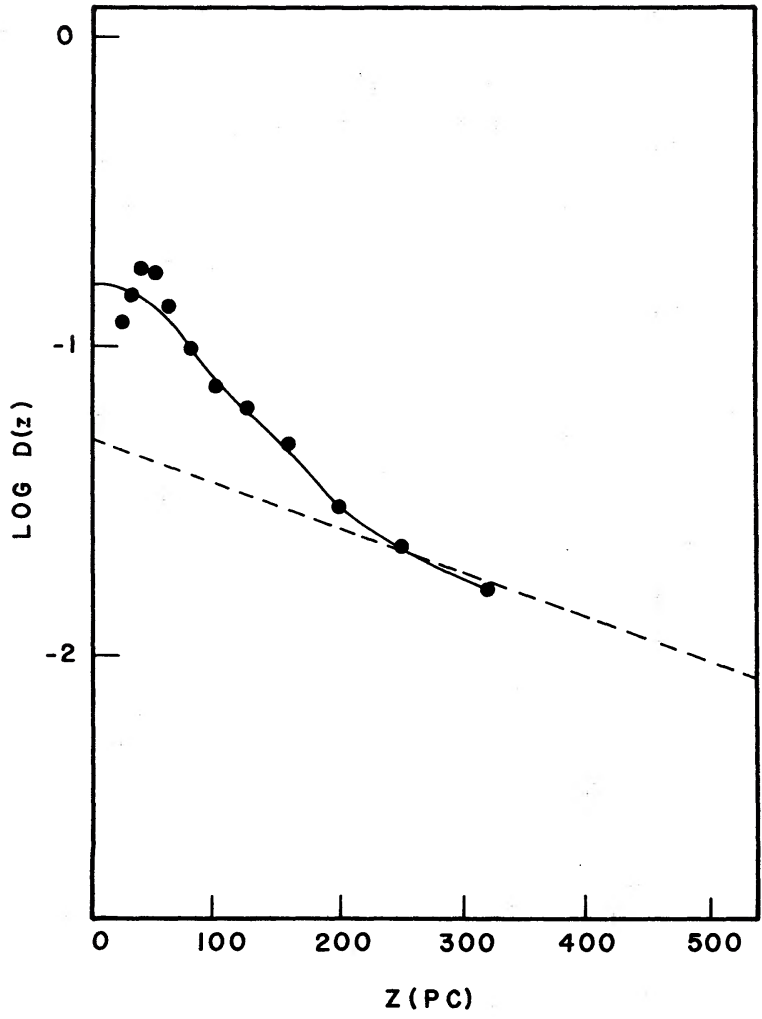

Fig. 7. Composite disk density distribution derived from dwarfs with $(B-V)_{0}$ greater than 1.4. The filled circles indicate computed points. The adopted smooth curve is given by the solid line. The Case I distribution, shifted vertically to fit the adopted curve, is indicated by the dashed line.

Fig. 5. For Case II, it is assumed that all stars are distributed according to the composite curve derived for the reddest stars, i.e., the solid curve with dashed extension of Fig. 7, normalized to $\log D(z)=0.00$ at $z=0$. In Case III, stars redder than $(B-V)_{o}=1.40$ are assumed to be distributed according to the normalized composite curve derived for these stars (solid curve with dashed extension in Fig. 7 normalized to $\log D(z)$ $=0.00$ at $z=0$ ), while the bluer stars are assumed to have the same density distribution as assumed for Case I, i.e., the dashed curve in Fig. 7 normalized to $\log D(z)=0.00$ at $z=0$.

\section{LUMINOSITY FUNCTIONS}

Given star counts for narrow intervals of color or absolute magnitude, and the relative density distributions, the stellar density in the plane for stars in each interval can be determined by fitting predicted counts [Eq. (2)] to observed counts. Using the parameters and composite density curves derived in previous sections, the star counts as a function of apparent magnitude were predicted for each $(B-V)_{o}$ interval, assuming unit density in the plane. The density in the plane was then derived by summing the predicted counts over apparent magnitude and scaling the total to the sum of the observed counts for the color interval. The luminosity functions so derived are shown in Figs. 8(a)8(c) for Cases I, II, and III. Also shown are the McCuskey (1956) and van Rhijn luminosity functions for comparison. The bump between absolute magnitudes 6 and 8 in the luminosity functions seems well established, as there are several points contributing to it. The existence of the decrease beyond absolute magnitude 8 is subject to doubt, however, as it is defined by one point only. The difference at faint magnitudes between the luminosity function derived here and that of van Rhijn can be attributed to the small number of intrinsically faint stars for which data were available to van Rhijn (van Rhijn 1925). It should be noted that the luminosity functions for Cases I and II just bracket the van Rhijn luminosity function for absolute magnitude brighter than 10 . This is not surprising, as the true density distribution might reasonably be expected to lie somewhere between Cases I and II. The most remarkable feature of the luminosity function is the steep rise beyond absolute magnitude 9 , which seems well established. As will be seen in the next section, this result has significant consequences for the mass in the neighborhood of the sun as determined by nondynamical means.

It is of interest to estimate the mass density in the solar neighborhood due to stars. For this purpose it is necessary to choose the best available representation of the luminosity function. The luminosity functions calculated here for stars brighter than $M_{V}=9$ vary as a function of the density distribution assumed close to the plane. With the present data we cannot distinguish between these functions. Acknowledging this uncertainty and noting that the Case I and Case II luminosity functions bracket the van Rhijn luminosity function, we adopt as the best representation of the luminosity function the van Rhijn function for stars brighter than $M_{V}=9.5$ and the Case III luminosity function for fainter stars.

TABLE VI. Local mass density of stars.

\begin{tabular}{|c|c|c|c|c|c|c|}
\hline \multirow[b]{2}{*}{$M_{V}$} & \multicolumn{6}{|c|}{ Mass Density $\left(10^{-2}\right.$ solar masses per $\left.\mathrm{pc}^{3}\right)$} \\
\hline & Case I & Case II & Case III & $\begin{array}{l}\text { van } \\
\text { Rhijn }\end{array}$ & $\begin{array}{c}\text { van } \\
\text { Rhijn-III }\end{array}$ & $\begin{array}{c}\text { van } \\
\text { Rhijn-I }\end{array}$ \\
\hline$\leq 2$ & & & & 0.27 & 0.27 & 0.27 \\
\hline-3 & 0.20 & 0.62 & 0.20 & 0.14 & 0.14 & 0.14 \\
\hline 4 & 0.16 & 0.53 & 0.17 & 0.23 & 0.23 & 0.23 \\
\hline 5 & 0.16 & 0.50 & 0.15 & 0.24 & 0.24 & 0.24 \\
\hline 6 & 0.21 & 0.68 & 0.21 & 0.23 & 0.23 & 0.23 \\
\hline 7 & 0.21 & 0.65 & 0.21 & 0.20 & 0.20 & 0.20 \\
\hline 8 & 0.12 & 0.35 & 0.12 & 0.22 & 0.22 & 0.22 \\
\hline 9 & 0.12 & 0.35 & 0.20 & 0.31 & $\underline{0.31^{\mathrm{a}}}$ & 0.31 \\
\hline 10 & 0.17 & 0.45 & 0.46 & 0.32 & $\overline{0.46}$ & $0.32^{\mathrm{a}}$ \\
\hline 11 & 0.38 & 0.86 & 0.89 & 0.32 & 0.89 & 0.38 \\
\hline 12 & 0.80 & 1.41 & 1.40 & 0.24 & 1.40 & 0.80 \\
\hline 13 & 1.60 & 2.10 & 2.08 & 0.18 & 2.08 & 1.60 \\
\hline Total & 4.1 & 8.5 & 6.1 & 2.9 & 6.7 & 5.0 \\
\hline
\end{tabular}

a Entries above line were computed using van Rhijn luminosity function. Others were computed using designated luminosity function from this study. 


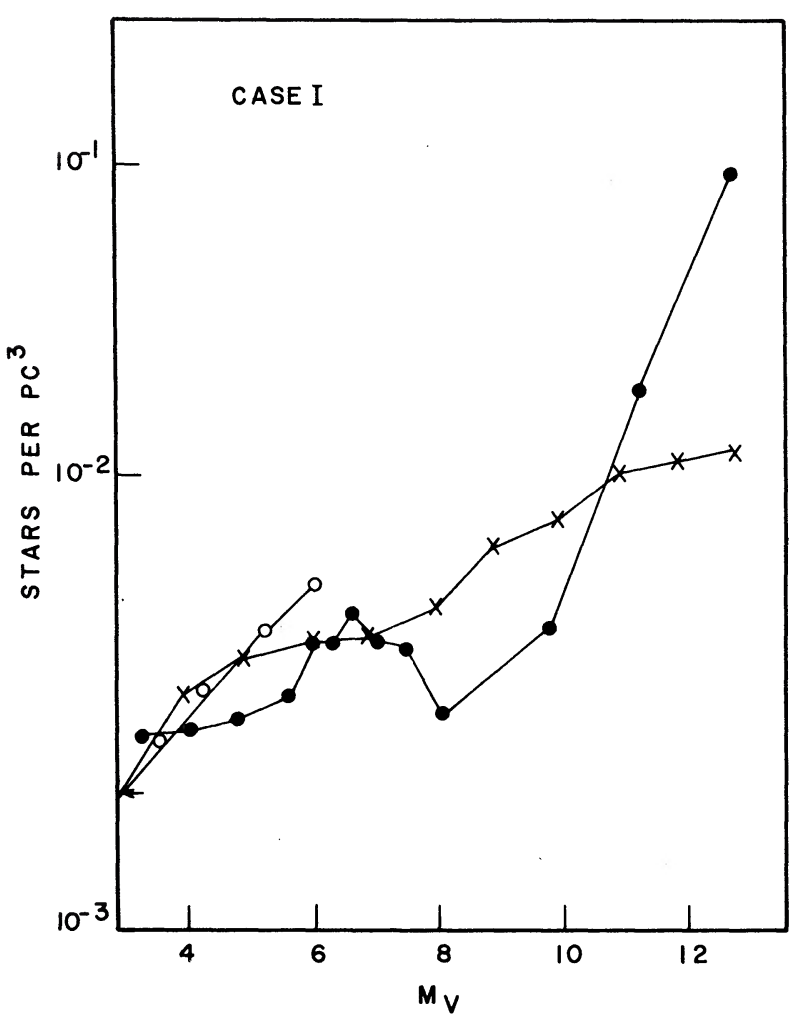

(a)

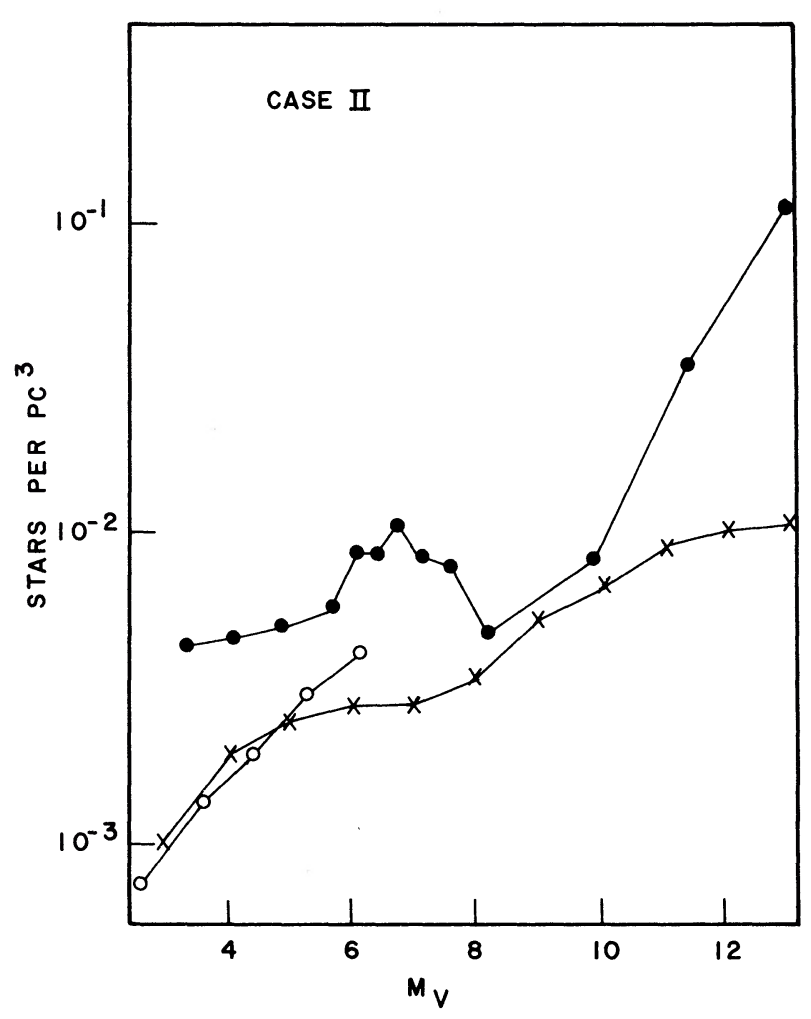

(b)

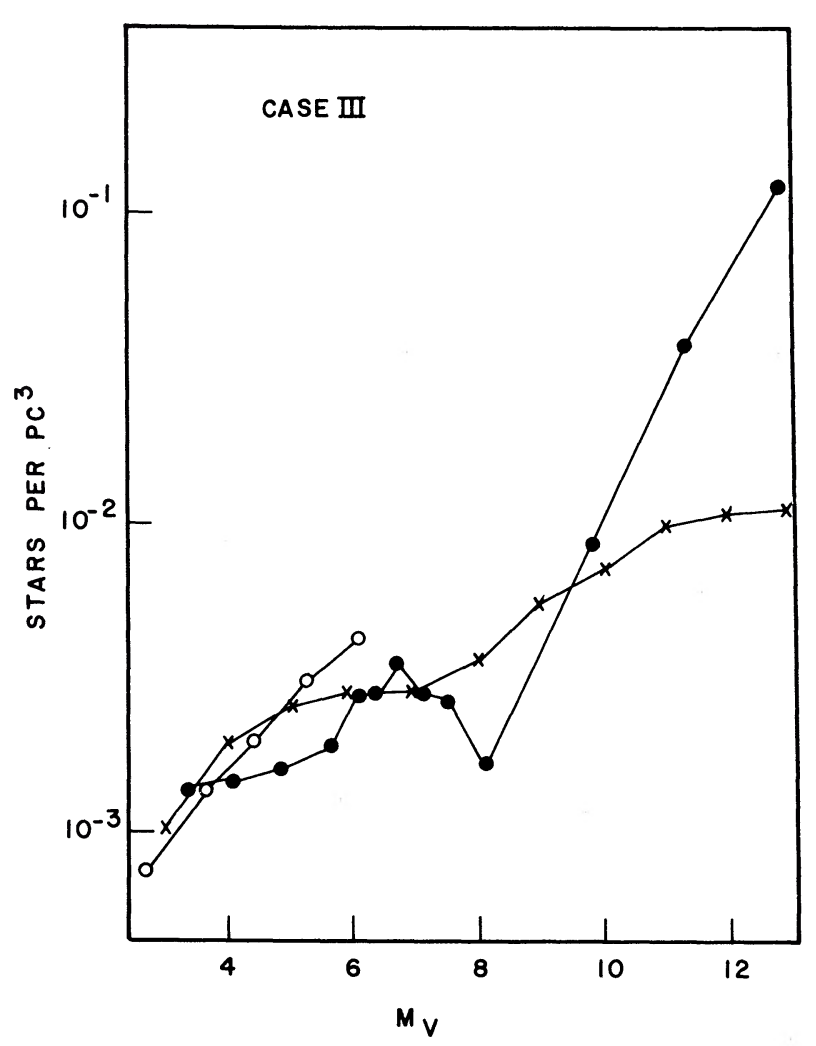

(c)

FIg. $8(\mathrm{a}-\mathrm{c})$. Computed luminosity functions ( $\bullet$ ). The van Rhijn ( $x$ ) and McCuskey (O) luminosity functions are shown for comparison. 


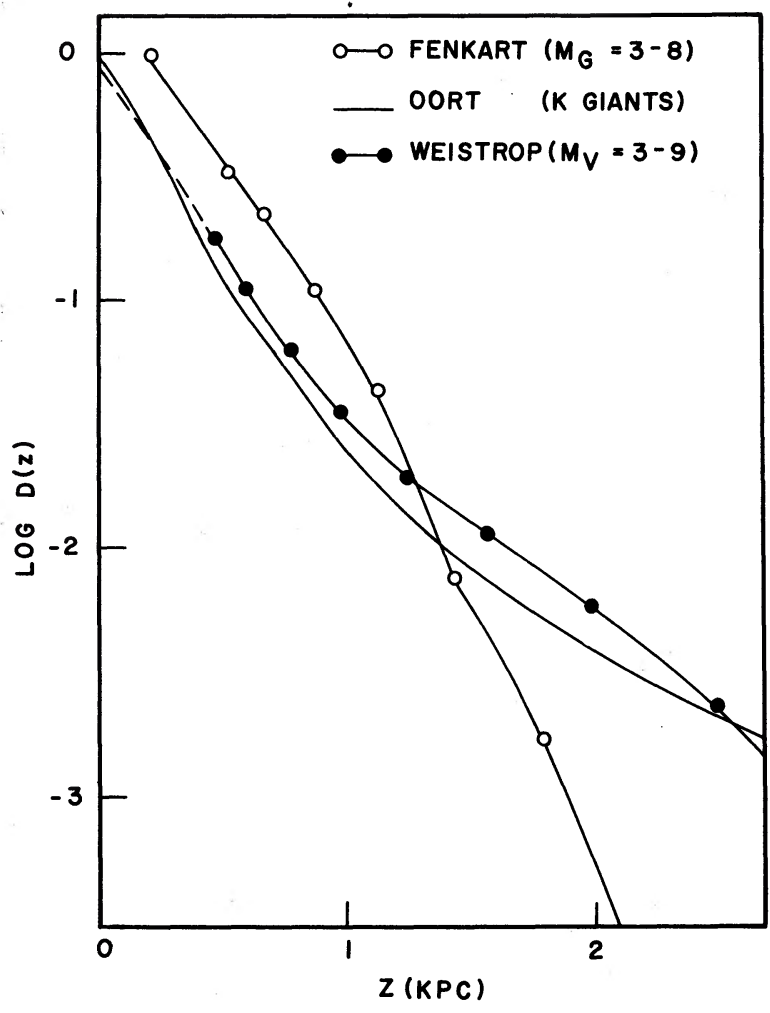

Fig. 9. Comparison of density distributions derived for disk stars. The kind of stars used to determine each curve is indicated after the author's name. (Dashed line indicates extrapolated portion of curve.)

The mass-luminosity relation adopted follows the formula given by Schmidt (1959), which agrees well with the data of Harris, Strand, and Worley (1963) and Eggen (1967) and adequately approximates the masses of the faintest stars, as reported by van de Kamp (1971). The result of applying the massluminosity relation to the luminosity functions for Cases I, II, and III are shown in Table VI. Also shown are the results for the van Rhijn luminosity function and two combined luminosity functions, consisting of van Rhijn's data for bright stars and the data derived for Case III for stars fainter than absolute magnitude 9.5, and van Rhijn's function plus the data for Case I for stars fainter than absolute magnitude 10.5 [see Figs. 8(a) and 8(c)]. The masses derived using the van Rhijn luminosity function include contributions for all stars fainter than $M_{V}=-5.0$. The differences among the various determinations reflect the differences in the luminosity functions assumed. The dominant contribution to the total mass is made by the large number of faint stars in all cases. The results for the models considered to be most likely (Case III and van Rhijn-Case III) indicate a mass density of between 0.06 and 0.07 solar masses per $\mathrm{pc}^{3}$ for the type of stars considered here.

\section{DISCUSSION}

Currently accepted values of the interstellar absorption at high-galactic latitude generally range between $A_{V}=0.00$ and $0.10 \mathrm{mag}$ (Philip 1968; Crawford and Barnes 1969; Sandage 1969; Slettebak, Wright, and Graham 1968; Peterson 1970; McClure and Crawford 1971; Philip and Tifft 1971). In order to evaluate the influence of $A_{V}$ on the calculations, all results were rederived assuming $A_{V}=0.00$ mag. The density distributions and luminosity functions computed under this assumption did not differ significantly from those computed assuming $A_{V}=0.10 \mathrm{mag}$. The results presented here are therefore independent of interstellar absorption over the range of values considered.

In Fig. 9 the Case I disk density distribution derived from stars with $M_{V}=3-9$ is compared with Oort's curve (1960) for $\mathrm{K}$ giants and Fenkart's curve (1967) for stars with $M_{G}=3-8$. The dashed line indicates the extrapolation adopted due to the uncertainty in $D(z)$ for small $z$, as described in Sec. II. Fenkart's curve is slightly high, due to the normalization at $250 \mathrm{pc}$ from the galactic plane. If Fenkart's result is forced to coincide with Oort's at $250 \mathrm{pc}$, the agreement with Oort is excellent to $z=1000$ pc. Beyond 1000 pc, Fenkart's curve falls off faster than either Oort's or the result found here. The density distribution derived here is consistent with the renormalized Fenkart curve to 1

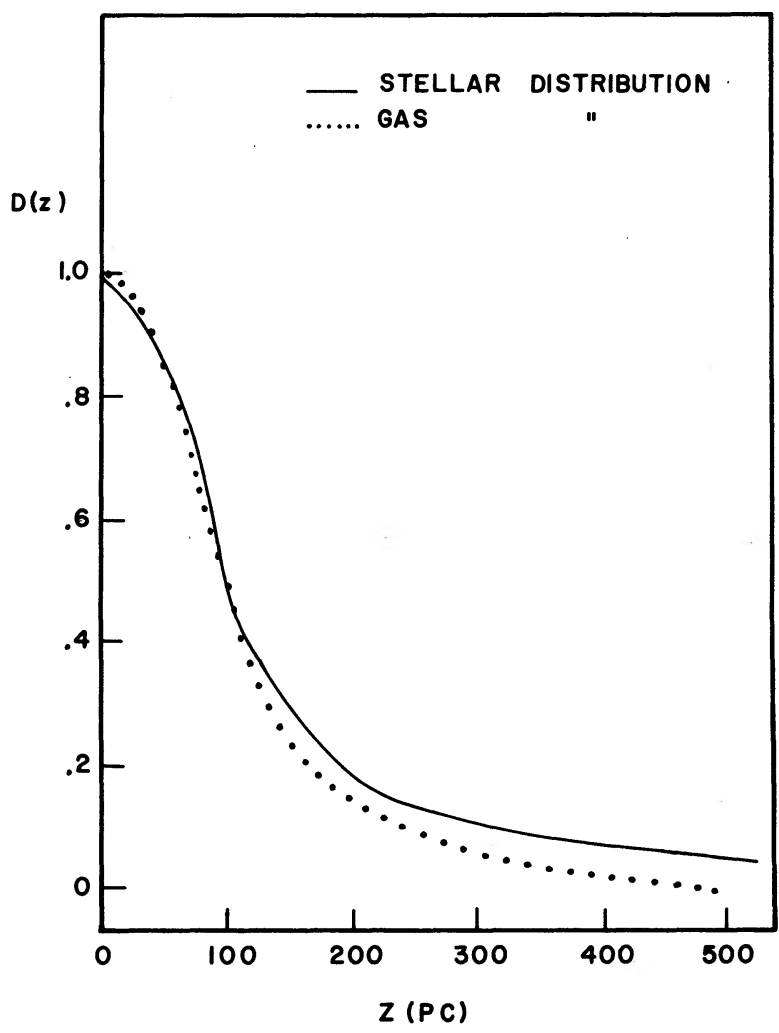

FIG. 10. Comparison of relative density distributions for intrinsically faint stars and gas. 


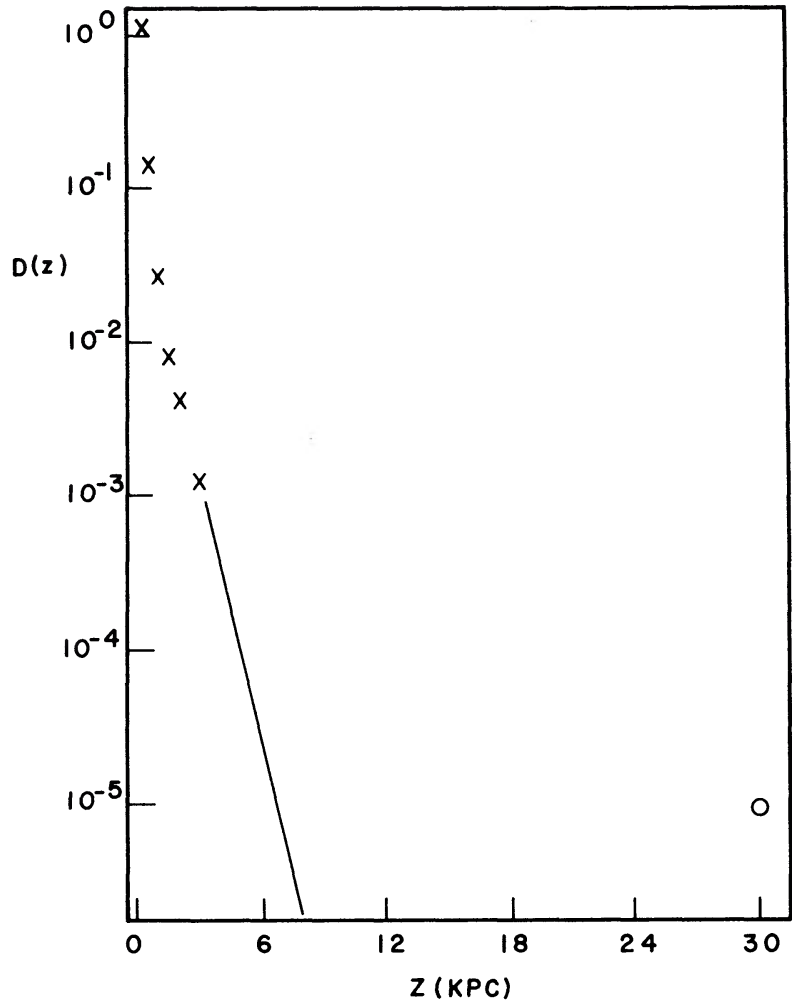

Fig. 11. Extrapolated Oort $\mathrm{K}$ giant density distribution. Points taken from Oort's curve are indicated by $(\boldsymbol{x})$. See text for explanation of $(0)$.

$\mathrm{kpc}$, and with Oort to $2 \mathrm{kpc}$. Considering the uncertainties in the data beyond $2 \mathrm{kpc}$, the agreement with Oort at those distances is not unsatisfactory. The discrepancy between the distribution we have derived and that of Fenkart lies in the different methods used to distinguish between Population II and disk stars. Using the ultraviolet excess as a population discriminant, Fenkart has assigned a larger fraction of the faint stars to the halo population than we have done (Paper I), producing the observed difference in the disk density curves.

The Case II curve is compared with the density distribution of the gas (Schmidt 1957) in Fig. 10. The agreement between the curves is good for $z$ less than $150 \mathrm{pc}$, and within the errors of the curves for larger $z$. From the figure it can be seen that the reddest stars, which define the inner part of the curve, are as highly concentrated to the plane as the gas. Figure 10 thus suggests the possibility that the intrinsically faint stars may in fact be very young stars, recently formed from the gas. However, the dispersion of the velocities perpendicular to the plane for red dwarfs does not indicate such a narrow layer. Using Eggen's data (1968) for stars selected without reference to velocity criteria, the velocity dispersion as a function of color was calculated (Table VII). As can be seen from the results, no narrowing of the layer occurs for stars bluer than $(B-V)_{o}=1.60$.
The number of redder stars is insufficient to determine the velocity dispersion with any accuracy. On the other hand, recent results of a study of the proper motions of 21 M dwarfs near the North Galactic Pole (Murray and Sanduleak 1972) indicate that the transverse velocity dispersion of these stars is small, and that they may indeed be concentrated to the galactic plane. It should also be noted that while a smooth, monotonically decreasing curve has been fitted to the Case II density distribution, as can be seen from Fig. 7, the individual points are not inconsistent with a peak in the density distribution at $z=50 \mathrm{pc}$, as suggested by Elvius (1965).

Before discussing the consequences of the luminosity function derived for disk dwarfs, the possibility that the star counts producing this result are in fact due to other types of stars will be considered. In order to account for the stars as Population II giants, the number of giants predicted to be at $z=100 \mathrm{kpc}$ [using the equation of stellar statistics, Eq. (2)] must be increased by a factor of one hundred to one thousand. Such an increase cannot be accomplished by a change in the Population II luminosity function, as the number of Population II stars predicted for $V=12-14$ would then be five times greater than the total number of stars observed. Nor can the star counts be reproduced by an increase in the Population II density distribution at large $z$, since any change sufficient to account for the observed number of stars with $(B-V)_{o}$ greater than 1.4, predicts more stars than were observed for bluer colors. If the maximum permissible changes in both the Population II luminosity function and density distribution are postulated, the observed star counts for $(B-V)_{o}$ $=1.6-1.7$ are still a factor of ten larger than can be accounted for by the Population II giants. The observations cannot be attributed to Population II dwarfs, either, as it is extremely unlikely that Population II stars are distributed in the very narrow layer that has been derived from the observed star counts. While the possibility that the observations may be due to disk giants cannot be definitely eliminated, the probability is small, owing to the high density of giants which would be required far from the galactic plane. Since we have tied the luminosity function of the giants to observations for $V=11-12$ (Sec. I), only a change in the density distribution can be used to fit the number of

TABLE VII. Velocity dispersion as a function of color.

\begin{tabular}{|c|c|c|}
\hline$B-V$ & $\begin{array}{c}\text { Disp } \\
Z \\
\mathrm{~km} \mathrm{sec}^{-1}\end{array}$ & No. Stars \\
\hline 1.05 & 21 & 58 \\
\hline 1.15 & 20 & 62 \\
\hline 1.25 & 23 & 65 \\
\hline 1.35 & 33 & 73 \\
\hline 1.45 & 19 & 68 \\
\hline 1.55 & 22 & 32 \\
\hline 1.65 & 5 & 6 \\
\hline 1.75 & 8 & 2 \\
\hline
\end{tabular}




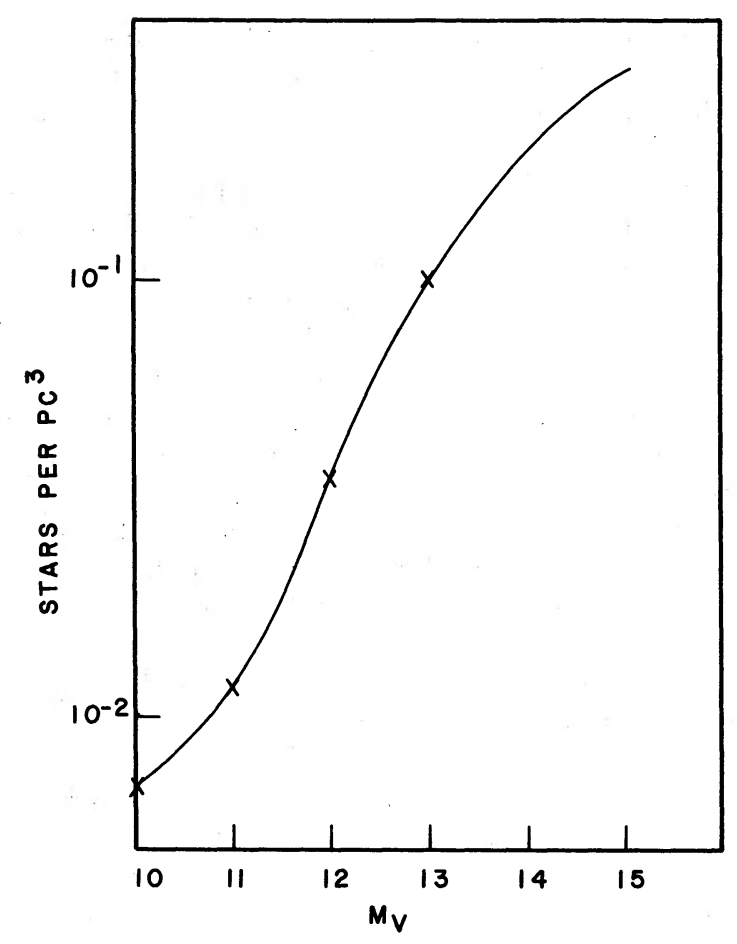

(a)

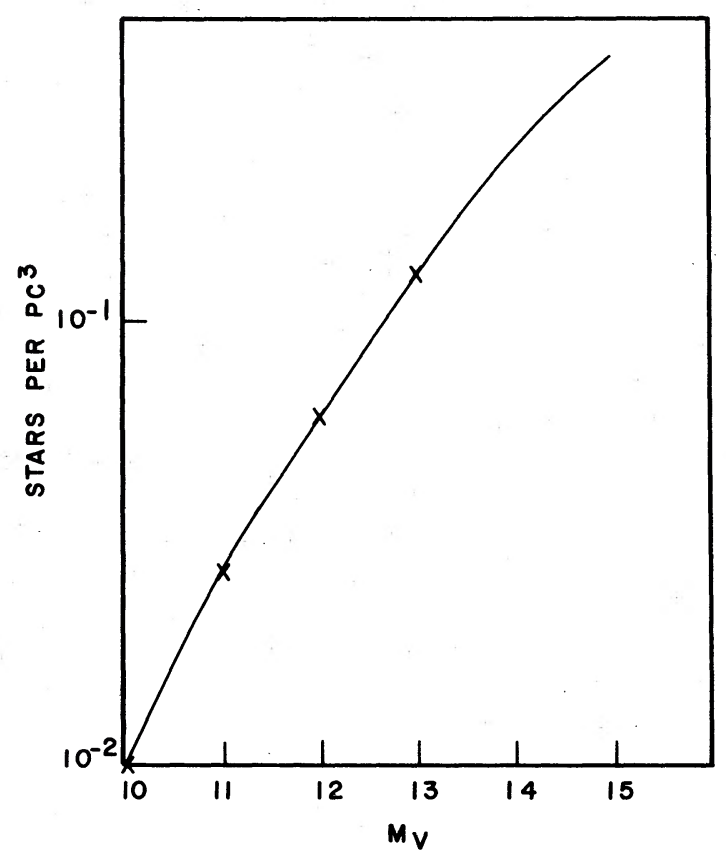

(b)

FIg. $12(a-b)$. Extrapolations of the combined luminosity functions, (a) van Rhijn-Case I, and (b) van Rhijn-Case III.

disk giants to the observations. The increase in density required to make that fit is indicated in Fig. 11, where the crosses represent the Oort giant density distribution, extrapolated as indicated by the solid line, and the circle at $30 \mathrm{kpc}$ indicates the density needed to reproduce the observations. As can be seen from the figure, the required density distribution cannot be definitely ruled out, but would assume a substantial flattening of the curve beyond $5 \mathrm{kpc}$. Because of these arguments, it seems most probable that the observations are in fact due to disk dwarfs, as has been assumed. In any event, the enhanced luminosity function of red dwarfs is not completely unexpected, as an investigation by Sanduleak, reported by Bok (1967), indicates a very large number of $M$ dwarfs in the range $11<M_{V}<16$. Moreover, Murray and Sanduleak (1972), in their recent study of $M$ dwarfs, suggest the existence in the solar neighborhood of a large number of these stars not previously discovered. They estimate the contribution of these stars to the local mass density to be of the order of 0.05 solar masses per $\mathrm{pc}^{3}$ for stars of $M_{V} \leq 13$. This value is in good agreement with the results we have reported (Table VI.)

Assuming the luminosity functions derived here, the local mass density calculated for disk stars is greater than that previously determined (Table VI). The increase in the mass density is due exclusively to the large number of intrinsically faint stars, except for the Case II result, which also contains an additional contribution from brighter stars. The combined van Rhijn-Case I and van Rhijn-Case III luminosity functions will be used to estimate the total mass near the sun due to disk stars. The red end of the van RhijnCase I function is shown in Fig. 12(a) with an extension drawn free hand to $M_{V}=15$. The extension has been drawn under the assumption that the luminosity function increases to $M_{V}=15$ at approximately the same rate as the increase from $M_{V}=11$ to 13. This assumption is supported by the preliminary results of Luyten (1968) which indicate that the luminosity function rises to $M_{p g}=+15.7$ and then turns over. Still, the extension used must be considered an upper limit, and the possibility that the luminosity function becomes flatter beyond $M_{V}=13$ cannot be excluded. We have terminated the function at absolute magnitude 15 , corresponding to a mass of 0.08 suns in the adopted massluminosity relation, because of recent theoretical arguments which indicate the minimum mass of a main sequence star to be about 0.08 to 0.10 solar masses (Straka 1971a, 1971b; Hoxie 1970). Table VIII shows

TABLE VIII. Extended luminosity functions and total mass densities.

\begin{tabular}{|c|c|c|c|c|}
\hline \multicolumn{3}{|c|}{ van Rhijn-Case I } & \multicolumn{2}{|c|}{ van Rhijn-Case III } \\
\hline$M_{V}$ & $\begin{array}{l}\text { Luminosity } \\
\text { function }\end{array}$ & $\begin{array}{c}\text { Mass } \\
\text { Density } \\
\text { (solar } \\
\text { masses } \\
\text { per pc } \text { pr) }^{3}\end{array}$ & $\begin{array}{l}\text { Luminosity } \\
\text { function }\end{array}$ & $\begin{array}{c}\text { Mass } \\
\text { Density } \\
\text { (solar } \\
\text { masses } \\
\text { per } \mathrm{pc}^{3} \text { ) }\end{array}$ \\
\hline$\leq 13$ & & 0.050 & & 0.067 \\
\hline 14 & 0.20 & 0.024 & 0.25 & 0.030 \\
\hline 15 & 0.30 & 0.024 & 0.40 & 0.032 \\
\hline \multirow{2}{*}{\multicolumn{2}{|c|}{ Other sources }} & 0.098 & & 0.129 \\
\hline & & 0.05 & & 0.05 \\
\hline \multicolumn{2}{|c|}{ Total } & 0.15 & & 0.18 \\
\hline
\end{tabular}


the total mass in the solar neighborhood contributed by stars, as calculated with the extended function. To this must be added 0.01-0.03 solar masses per $\mathrm{pc}^{3}$ for the white dwarfs (Weidemann 1969) and 0.010-0.025 solar masses per $\mathrm{pc}^{3}$ for the interstellar matter (Oort 1958; Westerhout 1957), for an addition of about 0.05 solar masses per $\mathrm{pc}^{3}$. From dynamical arguments, Oort (1960) has shown that the expected mass density in the solar neighborhood is 0.15 solar masses per $\mathrm{pc}^{3}$. From the table we see that all of that mass can now be accounted for, using the extrapolated luminosity function.

When considering the van Rhijn-Case III luminosity function, we must also take into account Oort's calculation (1965) that the amount of unknown mass in the plane is increased $40 \%$, to 0.21 solar masses per $\mathrm{pc}^{3}$, if it is distributed in a narrow layer. Figure 12(b) shows the van Rhijn-Case III luminosity function extrapolated free hand to $M_{V}=15$ in the same way that the van Rhijn-Case I function was extrapolated. Again, the last two values should be considered as indicating the upper limit of the luminosity function for stars fainter than $M_{V}=13$. The extended luminosity function and corresponding mass density are given in Table VIII. A total of 0.18 solar masses per $\mathrm{pc}^{3}$ can be accounted for using the extrapolated luminosity function. Thus, without making unreasonable extensions of the luminosity functions, it is possible to account for essentially all of the required mass in the solar neighborhood using either of the density distributions that have been considered. It should also be noted that stars fainter than $M_{V}=15.5$ do exist. Greenstein, Neugebauer, and Becklin (1970) call attention to W359 and VB10, with absolute visual magnitudes 16.6 and 18.9, respectively. Greenstein et al. consider these stars in defining the faint end of the main sequence. Whether they are in fact below the theoretical lower mass limit for main sequence stars depends principally on the mass-luminosity relation adopted. Greenstein et al. estimate masses of $\lesssim 0.1$ solar masses, while the relation adopted here would assign masses of 0.05 solar masses and 0.02 solar masses to these stars. Another faint star, Ross 614B $\left(M_{V}=16.8\right)$ is reported by van de Kamp (1971), who finds a mass of 0.07 solar masses for this star. Whether these stars are unstable or actually on the main sequence still seems open to question. They do suggest, however, the possibility that a significant amount of mass exists in stars fainter than $M_{V}=15.5$.

\section{SUMMARY}

A reasonably reliable density distribution to distances of $z=2500 \mathrm{pc}$ has been derived for the disk population. The density gradient close to the plane is steeper than that found in previous studies, but beyond $300 \mathrm{pc}$ the shape of the distribution agrees well with that derived by Oort from the $\mathrm{K}$ giants. The steep density distribution, which is indicated by the intrinsically faint stars, needs further investigation, perhaps by extending the study to still fainter stars, or determining velocities perpendicular to the plane for the stars in question. Part of the missing mass in the solar neighborhood has been found in the increased number of faint stars. By extending the observed luminosity function in a not unreasonable way, it is possible to account for most of the mass in the solar neighborhood, whether the unknown component is distributed in a narrow layer or as the brighter disk stars.

\section{ACKNOWLEDGMENTS}

The author wishes to thank Professor M. Schmidt for many helpful discussions during the course of this investigation. We are also grateful to Dr. Murray and Dr. Sanduleak for making their results available prior to publication. The work reported here was supported in part by a NASA Traineeship.

\section{REFERENCES}

Becker, F. 1940, Z. Astrophys. 19, 50.

Blaauw, A. 1963, Basic Astronomical Data, edited by K. Aa. Strand (U. of Chicago Press, Chicago), p. 383.

Bok, B. J. 1967, Trans. I.A.U. 13A, pp. 683, 699

Bok, B. J., and MacRae, D. A. 1941, Ann. N. Y. Acad. Sci. 42, Art. 2, 219.

Crawford, D. L., and Barnes, J. V. 1969, Astron. J. 74, 1008.

Eggen, O. J. 1967, Ann. Rev. A stron. Astrophys. 5, 105.

- . 1968, Astrophys. J. Suppl. 16, 49.

Eggen, O. J., and Sandage, A. R. 1964, A strophys. J. 140, 130.

- 1969 , ibid. 158, 669 .

Elvius, T. 1965, Galactic Structure, edited by A. Blaauw and M. Schmidt (U. of Chicago Press, Chicago), p. 41.

Fenkart, R. P. 1967, Z. Astrophys. 66, 390.

Gliese, W. 1956, Z. Astrophys. 39, 1.

Greenstein, J. L., Neugebauer, G., and Becklin, E. E. 1970, Astrophys. J. 161, 519 .

Halliday, I. 1955, ibid. 122, 222.

Harris, D. L. III, Strand, K. Aa., and Worley, C. E. 1963, Basic Astronomical Data, edited by K. Aa. Strand (U. of Chicago Press, Chicago), p. 273.

Hill, E. R. 1960, Bull. Astron. Inst. Neth. 15, 1.

Hoffleit, D. 1964, Catalogue of Bright Stars (Yale University Press, New Haven), 3rd ed.

Hoxie, D. T. 1970, Astrophys. J. 161, 1083.

Johnson, H. L., and Morgan, W. W. 1953, ibid. 117, 313.

Johnson, H. L., and Sandage, A. R. 1955, ibid. 121, 616.

Kinman, T. D. 1965, ibid. 142, 655.

Luyten, W. J. 1938, Publ. Astron. Obs. Univ. Minnesota 2, No. 7. -. 1968, Monthly Notices Roy. Astron. Soc. 139, 221.

Malmquist, K. G. 1936, Stockholms Obs. Medd. No. 26.

McClure, R. D., and Crawford, D. L. 1971, Astron. J. 76, 31.

McCuskey, S. W. 1956, Astrophys. J. 123, 458.

Murray, C. A., and Sanduleak, N. 1972 (submitted to Royal Astron. Soc.).

Oort, J. H. 1932, Bull. Astron. Inst. Neth. 6, 249

- 1958, Stellar Populations, edited by D. J. K. O'Connel (Vatican Obs., Vatican City), p. 415.

- 1960, Bull. Astron. Inst. Neth. 15, 45.

1965, Galactic Structure, edited by A. Blaauw and

M. Schmidt (U. of Chicago Press, Chicago), p. 455.

Peterson, B. A. 1970, Astron. J. 75, 695.

Philip, A. G. D. 1968 , ibid. 73, 1000.

Philip, A. G. D. and Tifft, L. E. 1971, ibid. 76, 567.

Rhijn, P. J. van 1925, Publ. Kapteyn Astron. Lab. Groningen No. 38.

Rhijn, P. J. van 1936, ibid. No. 47.

Rhijn, P. J. van, and Schwassmann, A. 1935, Z. Astrophys. 10, 161.

Sandage, A. R. 1957, Astrophys. J. 125, 435.

-. 1962, ibid. 135, 333.

- .1969, ibid. 157, 515 . 
Schmidt, M. 1957, Bull. A stron. Inst. Neth. 13, 247.

—. 1959, Astrophys. J. 129, 243.

Slettebak, A., Wright, R. R., and Graham, J. A. 1968, Astron. J.

$73,152$.

Straka, W. C. 1971a, Astrophys. J. 164, 125.

-. 1971b, ibid. 165, 109.

Strand, K. Aa., and Riddle, R. K. 1970, Publ. U. S. Naval Obs.

XX, Part IIIC

Upgren, A. R. 1962, Astron. J. 67, 37.
- - 1963, ibid. 68, 194.

van de Kamp, P. 1971, Ann. Rev. Astron. Astrophys. 9, 103.

Weidemann, V. 1969, Low-Luminosity Stars, edited by S. S. Kumar (Gordon and Breach Science Publishers, New York), 311.

Weistrop, D. 1972, Astron. J. 77, 366.

Westerhout, G. 1957, Bull. A stron. Inst. Neth. 13, 201.

Woolley, R., Epps, E. A., Penston, M. J., and Pocock, S. B. 1970, Royal Obs. Annals No. 5.

\title{
Four-Color and $\mathrm{H} \beta$ Photometry for Open Clusters. VIII. IC 4665
}

\author{
D. L. Crawford and J. V. Barnes \\ Kitt Peak National Observatory,* Tucson, Arizona 85717
}

(Received 29 August 1972)

\begin{abstract}
Photoelectric $u v b y \beta$ photometry of $45 \mathrm{~B}-$-, A-, and F-type stars contained in or near the cluster IC 4665 were obtained at the Kitt Peak National Observatory between 1964 and 1972 . Thirty-two of these stars appear to be cluster members. The average color excess, due to interstellar reddening, is $E(b-y)=0^{\mathrm{m}} \cdot 14$ and is slightly variable over the field. The true distance modulus was found to be $V_{0}-M_{V}=7 \mathrm{~m}$. 5 by several different methods. Systematic effects in the photometry due to rotational velocity differences from star to star do not appear to be present in the B-type stars, but are evident in the A-type stars. The cluster appears to be slightly younger than the Pleiades.
\end{abstract}

\section{INTRODUCTION}

$T$ HE open cluster IC $4665 \quad$ (R. A. $=17^{\mathrm{h}} 43^{\mathrm{m}} \cdot 7$, Dec. $\left.=+5^{\circ} 33^{\prime}, 1950\right)$ near $\beta$ Oph, lies somewhat above the galactic plane $\left(l=30^{\circ} .6, b=+17 \% 1\right)$. The earliest spectral type of a member star is $\mathrm{B} 4 \mathrm{~V}$. We have included the cluster in our program of four-color and $\mathrm{H} \beta$ photometry of open clusters and associations as it is rather similar to the Pleiades, but has a quite different distribution of stellar rotational velocity with spectral type (Abt and Chaffee 1967). The cluster has also been rather well studied, membership of stars is well-known, and sufficient data are available with which to compare our own.

Star identification numbers, positions, rough magnitudes, and spectral types have been published by Kopff (1943). Vasilevskis (1955), and Sanders and van Altena (1972) have determined proper motions and estimated membership on the basis thereof.

Abt and Snowden (1964) and Abt, Bolton, and Levy (1972) have published radial velocities of most of the member stars and of a number of the nonmembers. They show that it is difficult to isolate members from nonmembers solely on the basis of radial velocities. Data of the latter paper show that 18 of the 19 brightest member stars are spectroscopic binaries.

$U B V$ photometry has been published by $\mathrm{H}$. L. Johnson (1954), Hogg and Kron (1955), Alcaino (1965), and McCarthy and O'Sullivan (1969), the latter being mostly photographic $V$ and $(B-V)$. In this paper we

\footnotetext{
* Operated by the Association of Universities for Research in Astronomy, Inc., under contract with the National Science Foundation.
}

have used the Hogg and Kron (1955) values, as their list of photoelectric measures is most complete. In a few cases, where they did not obtain $(U-B)$ values, we have used $(U-B)$ values of Alcaino (1965), after a correction of $+0^{\mathrm{m}} .02$, which is the average difference between all other photoelectric $(U-B)$ values in the two papers.

A number of the papers give a rather good description of the cluster properties and refer to other, mostly earlier, work on it. Suffice to summarize here that the cluster is quite similar to the Pleiades in its earliest spectral type (and hence, in age, probably), but has a significantly different percentage of binaries (IC 4665 has many, the Pleiades few), and the B-type cluster members have lower rotational velocities at a given spectral type. In addition, the main sequence in IC 4665 appears to stop on the faint end at about F5V $\left(M_{V} \simeq+3^{\mathrm{m}} \cdot 5\right)$ based on the proper motion studies and on star counts in the cluster field and in adjacent regions.

We have observed essentially all stars listed as members by any of the authors above, as well as a number of nonmembers (primarily with late-type spectra) so that the use of the cluster as a "standard photometric region," in $U B V$, may be extended, if desired, to the $u v b y \beta$ systems.

\section{THE OBSERVATIONS}

We have obtained photoelectric uvby and $\mathrm{H} \beta$ photometry on stars in the general field of the cluster since the early 1960s. As most of the earliest measures were with nonstandard filters, we have omitted them from any consideration, and have used only the data obtained 